\title{
The subtalar and talonavicular joints: a way to access the long-term load intake using conventional CT-data
}

\author{
Fabian Mueller • Sebastian Hoechel • \\ Joerg Klaws • Dieter Wirz • Magdalena Müller-Gerbl
}

Received: 14 April 2013/Accepted: 5 September 2013/Published online: 20 September 2013

(C) Springer-Verlag France 2013

\begin{abstract}
Purpose The aim of this study was to investigate the distribution of density of the subchondral bone plate within the articular surfaces of the subtalar and talonavicular joint regarding to its mineralisation and to verify whether a correlation to the mechanical bone strength exists.

Methods A total of 21 cadaverous lower leg specimens were investigated. Computed tomography osteo-absorptiometry (CT-OAM) was used to display the mineralisation of the subchondral bone plate analysing its density. The mechanical strength was measured by means of indentation testing. The distribution pattern was analysed regarding their dissemination with the main focus on number and location of their maxima. The correlation of both parameters was evaluated by linear regression.

Results The mineralisation and the mechanical strength were not distributed homogenously throughout the articular surfaces but showed unique and reproducible patterns. The range of absolute values for density and strength varied in between the samples and joint surfaces, but the number and location of the maxima evaluated by both methods showed to be concurring. The coefficient of correlation of both datasets ranged from 0.76 to 0.95 (median 0.88) and showed a linear dependency.

Conclusions Density distribution and mechanical strength of the subchondral bone plate are significantly associated and can be seen as a mirror of the long-term load
\end{abstract}

F. Mueller · S. Hoechel $(\bowtie) \cdot$ J. Klaws $\cdot$ M. Müller-Gerbl Institute of Anatomy, University of Basel, Pestalozzistrasse 20, 4056 Basel, Switzerland

e-mail: sebastian.hoechel@unibas.ch

D. Wirz

Laboratory of Biomechanics and Biocalorimetry, University of Basel, Klingelbergstrasse 50-70, 4056 Basel, Switzerland intake of a joint. It can be concluded that CT-OAM as a tool to visualize subchondral bone plate density distribution regarding to its mineralisation can be used to indirectly gain information about joint biomechanics in vivo by the use of conventional CT-data.

Keywords Mineralisation - Mechanical strength · Subchondral bone plate - CT-OAM .

Subtalar and talonavicular joint · Bone density

\section{Introduction}

The subtalar and the talonavicular joint are among the most complex weight-bearing joints of the human body and are highly variable in size and shape [7, 25, 26]. Even minor biomechanical or anatomical details may have considerable clinical significance [9] as these joints perform static, kinematic, and dynamic functions. On one hand, they distribute the weight of the body onto the foot; on the other hand, they serve as a rotatory force converter and reduce the stress acting on the ankle joint [19]. In connection with the bones of the forefoot these joints allow the adaption to uneven ground when walking or running.

The subchondral bone plate (SBP) is seen as a linkage between the cartilage and the cancellous bone of a joint [12]. Its main function is to transmit the impact forces acting on the cartilage and to disperse them on the underlying trabecular structures [15]. This long-term load intake leads to specific mineralisation patterns within the SBP which can therefore be regarded as loading history of an articular surface. These unique mineralisation patterns are mainly dependent on the joint situation itself defined by the shape and the geometry and individual factors like age and activity [18]. 
Changes in structure and density of the SBP are associated with the development and progression of osteoarthritis. By increasing the mechanical stiffness of the SBP, more energy is being transferred through the overlying cartilage which results in cartilage degeneration [20]. In general, the SBP and the cartilage can be considered as a functional unit, in which any modification of either of these two will entail remodelling of the other [13]. These findings are further supported by the work of Akiyama et al. [2]; for the subtalar joint it could be shown that the regions of greatest cartilage thickness correspond to the regions where the mineralisation of the SBP shows the highest density. This makes it even more important to detect changes in the mineralisation of the SBP in early stages to take precautionary steps and thus to avoid increasing damage.

Information about the mineral distribution within the SBP can in vivo be gained with the technique of computed tomography osteo-absorptiometry (CT-OAM). In contrast to conventional methods of CT-densitometry which measure the concentration of calcium hydroxyl apatite over a larger area, CT-OAM is a procedure to display regional differences in mineralisation within a whole joint surface [18].

Previous studies investigating the load on the subtalar and talonavicular joint mainly based on static conditions which represent the load transfer at time but leave out conclusions about the long-term distribution. By means of pressure-sensitive film the instantaneous load on the articular surfaces was determined [14, 21, 23, 30]. To describe the long-term load intake, CT-OAM can be used on conventional CT-data to get a deeper insight in the changes of the mineralisation distribution in the subtalar and talonavicular joint caused by long-term stress.

Recently, the mineralisation and mechanical strength of the SBP was investigated on the shoulder joint, the inferior tibial facies, and the patellofemoral joint. It could be shown that CT-OAM can be used to indirectly gain information about the mechanical strength of the SBP $[10,16]$.

The purpose of the present study was (1) to investigate the density distribution within the articular surfaces of the subtalar and talonavicular joint (2) to measure the mechanical strength of the SBP by means of an osteopenetrometer, and (3) to verify whether a correlation of subchondral bone density and mechanical strength exists.

\section{Materials and methods}

\section{Preparation}

The study included a total of 21 lower leg specimens with available CT-datasets that were derived from cadavers obtained from the Department of Anatomy, Ludwig-
Maximilian University of Munich ( $n=16,10$ female, 6 male), and the Department of Anatomy, University of Fribourg ( $n=5,2$ female, 3 male), all embalmed in formaldehyde (4\%). All samples represent European adult bones. Patient age distribution was 61-95 years with an average of 80.4 (Table 1). The samples consisted of calcaneus, talus, and navicular bone. They were dissected free of soft tissue, the joint capsules were opened and the joints disarticulated. After photographic documentation, the samples were classified according to their anatomical structure of articular surfaces [7, 26]. As expected and prior seen in the literature, the size and shape of the articular surfaces showed a high variation (Table 2). Due to the technical limitation of indentation testing (IT) and the needed distance in between the measuring points, 4 samples (Classification Sharada et al. [26]: Grade 2d and 3) proved to be not suitable for IT of the anterior and middle articular surface of the talocalcaneal joint. For the analysis of the correlation of density (CT-OAM) and mechanical strength (IT), we therefore only investigated the posterior talocalcaneal joint as well as the talonavicular joint. The density distribution will be evaluated for all articular surfaces. No anthropometric data were obtained.

\section{Anatomical structure of the articular surfaces}

The samples were classified by Bunning and Barnett's [7] classification and the adapted classification of Sharada et al. [26]. On each articular surface present, the longest diameter $(x)$ was measured using the software VGStudio ${ }^{\circledR}$ Max 2.2 (Heidelberg, Germany). The second measured dimension (y) was defined perpendicular (Fig. 1).

To establish the configuration of the posterior articular surface of the talocalcaneal joint, we analysed the medial side of the posterior facet on the calcaneus and talus as described by Shahabpour et al. [25].

\section{CT-osteo-absorptiometry}

The CT-data required to display mineralisation were generated on a conventional CT-scanner (SOMATOM 16, Siemens, Erlangen, Germany, $120 \mathrm{kV}, 180 \mathrm{mAs}$, slice thickness $0.6 \mathrm{~mm}$, sagittal slices) at the Department of Anatomy, University of Basel. The CT-data were edited using the image analysing system ANALYZE 11.0 (Biomedical Imaging Resource, Mayo Foundation, Rochester, MN, USA). In a first step, 3D reconstructions of each bone were generated and displayed in a perpendicular view to the joint surfaces (Fig. 2a-d). To achieve the required view on all the articular surfaces, it was necessary to generate two 3D reconstructions of the talus, one in frontal, and one in transversal view (Fig. 2b, c). Next, the articular surfaces were cut from the 3D 
reconstructions. By means of "maximum intensity projection", the maximum density values to a depth of $3 \mathrm{~mm}$ of the underlying bone plate were projected onto the top surface for every single image point. To achieve better visualisation of density distribution the grey values were assigned to false-colours, where the highest density values $(>1,200 \mathrm{HU})$ were shown in black, lower values in red, yellow, green and blue (in descending order). The final step comprised superimposing the false-colour representation onto the 3D models which resulted in a presentation that showed the mineral distribution of the whole joint surface in accordance to topographical landmarks (Fig. 2e-h) and served as a basis for further investigation. To allow a comparison of the individual mineralisation patterns, a coordinate system $(15 \times 15$ units $)$ was projected onto each articular surface. In respect to the orientation of the bones in anterior and posterior position, the borders of the coordinate system matched the borders of the articular surfaces (Fig. 2e-h). This enabled a standardised localisation of the maxima of mineralisation. The coordinates of the maxima of mineralisation were noted and transferred into separate summation charts for each articular surface. The summation charts were divided into nine squares, each of them representing $5 \times 5$ units of the previously used coordinate system (Fig. 2i-q).
Indentation testing

According to the variations of shape and size of the middle and anterior talocalcaneal articular surfaces, IT was not possible here. The articular surfaces of the posterior talocalcaneal joint and the talonavicular joint where in preparation evaluated in size according to the measurements in $(x)$ and (y) dimensions, and divided into a standardised grid. Due to the differences in size, the number of measuring points had to be adapted to the investigated surface (calcaneus: posterior talar articular surface 10-15, talus: posterior calcaneal articular surface 11-15, navicular articular surface 13-17; navicular bone: talar articular surface 8). The minimal distance to the border of the articular surfaces and between two measuring points had to be between 7 and $10 \mathrm{~mm}$. For IT the samples were cemented in small plastic boxes [fixed with poly(methylmetacrylate) (PMMA)] and attached to a lockable and rotatable ball joint that allowed perpendicular measurements for each test point. IT was performed with a mechanical test machine, an osteo-penetrometer (Synergie 100, $2 \mathrm{kN}$ loadcell, MTS Systems, Eden Prairie, MN, USA), equipped with a steel needle (radius $1.3 \mathrm{~mm}$, area $5.3 \mathrm{~mm}^{2}$ ) with a conical tip. This way, frictionless testing without compaction of bone was achieved. For each

Table 1 Sample classification and maxima distribution of the posterior talocalcaneal joint

\begin{tabular}{|c|c|c|c|c|c|c|}
\hline Sample & Age (years) & Sex & Side & Density (8-bit HU) & Penetration force $(N)$ & $r^{2}$ \\
\hline 1 & 87 & M & Left & $116-210$ & $72-1,341$ & $0.84 *$ \\
\hline 2 & 81 & M & Right & 88-198 & $22-485$ & $0.86^{*}$ \\
\hline 3 & 75 & $\mathrm{~F}$ & Left & 94-189 & $37-543$ & $0.94 *$ \\
\hline 4 & 91 & $\mathrm{~F}$ & Right & $105-184$ & $23-457$ & $0.86^{*}$ \\
\hline 5 & 81 & $\mathrm{~F}$ & Left & $95-182$ & $15-487$ & $0.92 *$ \\
\hline 6 & 61 & $\mathrm{~F}$ & Right & 94-194 & $32-669$ & $0.90 *$ \\
\hline 7 & 85 & $\mathrm{~F}$ & Right & 94-186 & $27-458$ & $0.80 *$ \\
\hline 8 & 86 & M & Right & 93-196 & $55-724$ & $0.84 *$ \\
\hline 9 & 87 & $\mathrm{~F}$ & Right & $113-211$ & $99-1,269$ & $0.87 *$ \\
\hline 10 & 78 & $\mathrm{~F}$ & Left & $104-193$ & $21-630$ & $0.94 *$ \\
\hline 11 & 91 & $\mathrm{~F}$ & Left & 123-198 & $73-1,235$ & $0.76^{*}$ \\
\hline 12 & 72 & M & Left & $108-229$ & $58-1,106$ & $0.93 *$ \\
\hline 13 & 88 & $\mathrm{~F}$ & Right & $106-182$ & $22-616$ & $0.90 *$ \\
\hline 14 & 76 & M & Left & $112-205$ & $84-1,085$ & $0.95^{*}$ \\
\hline 15 & 79 & M & Left & 95-198 & 30-939 & $0.90 *$ \\
\hline 16 & 95 & $\mathrm{~F}$ & Left & $101-192$ & $30-567$ & $0.89 *$ \\
\hline 17 & 83 & $\mathrm{~F}$ & Right & $90-205$ & $17-1,058$ & $0.94 *$ \\
\hline 18 & 78 & $\mathrm{~F}$ & Left & $98-173$ & $9-167$ & $0.85^{*}$ \\
\hline 19 & 63 & M & Left & $109-183$ & $126-670$ & $0.78 *$ \\
\hline 20 & 85 & M & Right & 103-201 & $55-1,039$ & $0.92 *$ \\
\hline 21 & 67 & M & Left & $101-202$ & $33-874$ & $0.93 *$ \\
\hline
\end{tabular}

$* p<0.01$ 
Table 2 Clinical and statistical data of the specimens M male, F female

\begin{tabular}{|c|c|c|c|c|c|}
\hline \multirow[t]{2}{*}{ Sample } & \multicolumn{2}{|c|}{ Maxima distribution } & \multirow[t]{2}{*}{ Bunning's classification [7] } & \multirow{2}{*}{$\begin{array}{l}\text { Classification } \\
\text { Sharada et al. [26] }\end{array}$} & \multirow{2}{*}{$\begin{array}{l}\text { Configuration of the subtalar } \\
\text { joint Shahabpour et al. [25] }\end{array}$} \\
\hline & Calcaneus & Talus & & & \\
\hline 1 & M & M & A & $2 \mathrm{c}$ & 0 \\
\hline 2 & M & M & B & $1 \mathrm{a}$ & 3 \\
\hline 3 & M & M & A & $2 \mathrm{c}$ & 2 \\
\hline 4 & M & M & A & $2 \mathrm{~d}$ & 1 \\
\hline 5 & M & M & A & $2 \mathrm{a}$ & 0 \\
\hline 6 & M & M & A & $2 \mathrm{a}$ & 0 \\
\hline 7 & M & $\mathrm{B}$ & A & $2 \mathrm{a}$ & 3 \\
\hline 8 & M & $\mathrm{B}$ & A & $2 \mathrm{a}$ & 2 \\
\hline 9 & M & M & A & $2 b$ & 2 \\
\hline 10 & B & B & A & $2 \mathrm{~d}$ & 3 \\
\hline 11 & $\mathrm{~T}$ & B & A & $2 c$ & 4 \\
\hline 12 & $\mathrm{M}$ & M & A & $2 b$ & 1 \\
\hline 13 & $\mathrm{~T}$ & M & A & $2 b$ & 1 \\
\hline 14 & B & $\mathrm{M}$ & B & $1 \mathrm{a}$ & 2 \\
\hline 15 & $\mathrm{M}$ & B & A & $2 \mathrm{~d}$ & 0 \\
\hline 16 & B & M & B & 3 & 3 \\
\hline 17 & $\mathrm{M}$ & $\mathrm{M}$ & A & $2 c$ & 0 \\
\hline 18 & $\mathrm{~T}$ & $\mathrm{M}$ & A & $2 b$ & 1 \\
\hline 19 & $\mathrm{~T}$ & $\mathrm{M}$ & B & $1 b$ & 4 \\
\hline 20 & $\mathrm{M}$ & B & A & $2 c$ & 0 \\
\hline 21 & $\mathrm{M}$ & M & A & $2 a$ & 2 \\
\hline
\end{tabular}

$M$ monocentric, $B$ bicentric, $T$ tricentric
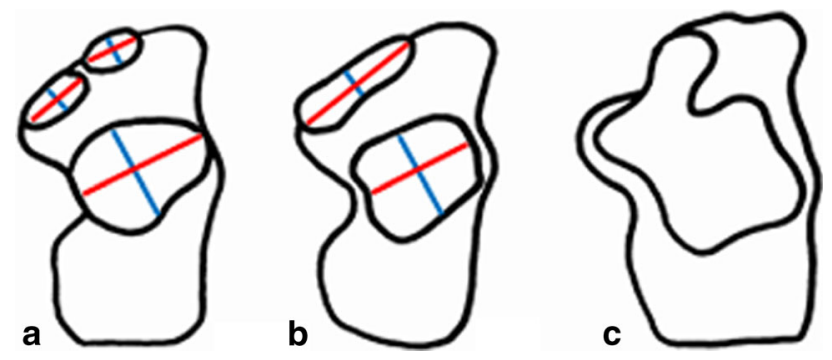

Fig. 1 The three types of calcanei according to Bunning's classification. a Bunning's Type A. b Bunning's Type B. c Bunning's Type C. Measured diameters on articular surfaces: $(x)$ red, $(y)$ blue. Adapted from Bunning and Barnett [7]

indentation point the test machine created a standardised hole with a depth of $7 \mathrm{~mm}$ at a speed of $1 \mathrm{~mm} / \mathrm{s}$. Simultaneously, the operating software recorded all occurring reactive forces in Newton (N) (Fig. 3). Afterwards, according to the procedure based on the work of Aitken et al. [1], Hoffmann et al. [11], and Saitoh et al. [22], the force maximum for each measuring point was identified and noted in a spread sheet.

\section{Density-strength correlation}

To calculate a possible correlation between density and strength, the corresponding density value (HU converted in 8-bit HU [10]) for each test point was measured. Using the 3D densitograms as a basis, the same test points as used for IT were identified by means of ANALYZE 11.0. To obtain the most accurate results, the measured area matched the surface of the indentation needle pin.

\section{Statistical analysis}

To evaluate statistical correlation between density and strength, linear regression was used. For all measured samples the Pearson product-moment correlation coefficient was established. Using a two-tailed $t$ test, we showed the significance $(p<0.01)$. For analysis of data distribution the Kolmogorov-Smirnov test was used. All statistical analyses were done using RStudio (RStudio: Integrateddevelopment environment for R, Version 0.96.122, Boston, MA, USA). 

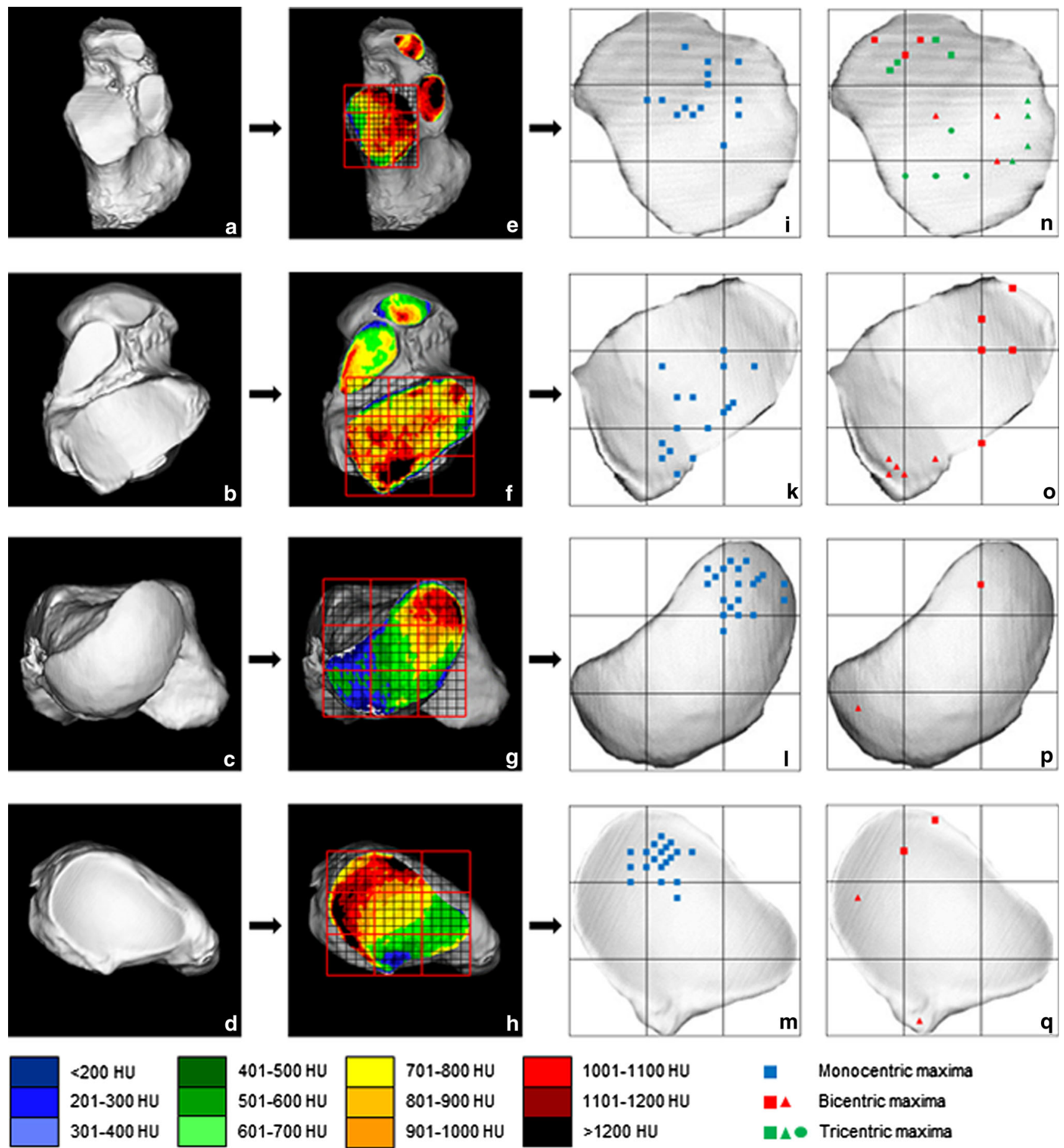

Fig. 2 Localisation of density maxima. a Calcaneus, superior aspect. b Talus, inferior aspect. c Talus, anterior aspect. d Navicular bone, posterior aspect. a-d Three-dimensional reconstructions for each bone, displayed in a perpendicular view of the joint surfaces. $\mathbf{e}-$ h Density distribution patterns generated by CT-OAM, overlaid by

the coordinate system used for standardised localisation of mineralisation maxima. $\mathbf{i}-\mathbf{m}$ Schematic representation of monocentric mineral maxima. $\mathbf{n}-\mathbf{q}$ Schematic representation of bicentric and tricentric mineral maxima 


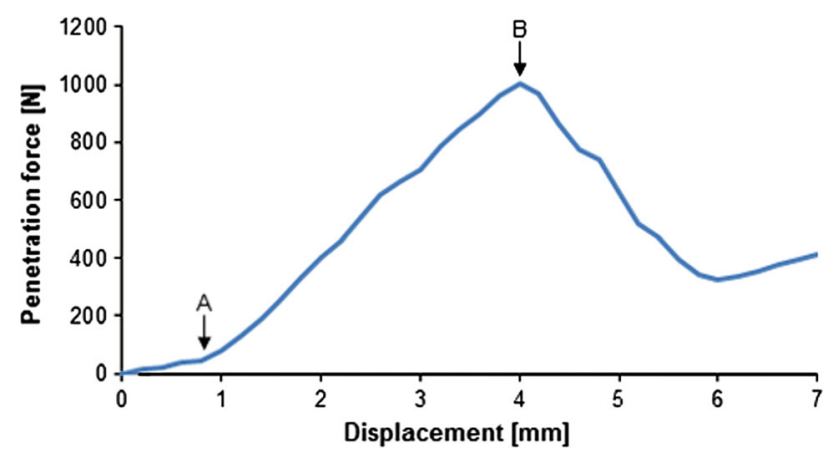

Fig. 3 Load deflection curve of indentation testing. At point $A$, the intender set contact with the SBP. The linear part of the curve up to point $B$ represents the stiffness of the sample. Point $B$ corresponds to the ultimate strength of the sample before fracture (maximal penetration force)

\section{Results}

Anatomical structure of the articular surfaces

Sample classification as seen in Table 2. Bunning's Type A $81 \%$, Type B, respectively, $19 \%$ of samples.

Measurements of the analysed articular surfaces are: Bunning's Type A: posterior articular surface of calcanei: (x) 29-38 mm; (y) 20-25 mm, middle articular surface of calcanei: (x) 16-24 mm; (y) 10-15 mm, anterior articular surface of calcanei: $(x)$ 9-15 mm; (y) 5-11 mm. Bunning's Type B: fused anterior and middle articular surface of calcanei: (x) 11-33 mm; (y) 10-13 mm.

Articular surfaces of the tali: posterior articular surface: (x) 19-25 mm; (y) 29-44 mm, middle articular surface: (x) 11-35 mm; (y) 10-17 mm, anterior articular surface: (x) 15-22 mm; (y) 7-17 $\mathrm{mm}$, articular surface for the navicular bone: $(x)$ 42-54 mm; (y) 20-30 mm.

Articular surface of the navicular bone for talus: (x) 18-36 mm; (y) 17-27 mm (Fig. 1).

\section{Subchondral mineralisation}

The results showed that the mineralisation of the SBP was not distributed homogenously as there were areas with high and low density values. From areas with high density values a steady decrease towards the periphery could be seen.

The density maxima found on the anterior articular surface of the tali showed to be monocentric and consistently on the medial part, for the middle one as well monocentric and located in the posterolateral area. The majority ( $n=15$ ) of the posterior articular surfaces of the tali presented a monocentric pattern with a central or posterolateral density maximum. The remaining specimens $(n=6)$ showed bicentric maxima; these were situated in the posteromedial and lateral portions of the articular surfaces (Figs. 2, 4; Table 2).

The density maxima found on the anterior articular surface (Bunning's Type A) of the calcanei were located in the posterior area. The middle articular surface presented the density maximum in the posterolateral part (Fig. 4). For Bunning's Type B the maximum was located laterally along the whole surface. The majority $(n=14)$ of the posterior articular surfaces showed a monocentric pattern with a central $(n=8)$, medial $(n=3)$, or anterior $(n=3)$ density maximum. Three specimens showed a bicentric pattern with anterior and medial maxima. Also, there could be found four tricentric patterns; their maxima were located at the anterior, the posterior, and the medial part of the articular surface (Figs. 2, 4; Table 2). The vast majority $(n=20)$ of the navicular articular surfaces of the tali showed a monocentric pattern; their maxima could be found dorsolaterally. Only one specimen showed a bicentric pattern whose maxima were located in the dorsolateral and caudomedial parts (Fig. 2).

The talar articular surfaces of the navicular bones showed monocentric $(n=19)$ and bicentric $(n=2)$ mineralisation patterns; the monocentric maxima could be found in the cranial or craniolateral, the bicentric maxima in the cranial, the lateral, and the caudal parts of the articular surfaces (Fig. 2).

The interindividual range of the maximal density values across all specimens was between $88 \mathrm{HU}$ and $211 \mathrm{HU}$ (8bit scale) (Table 1).

\section{Indentation}

IT showed a non-homogenous distribution of mechanical strength. The maxima were located in similar areas as the corresponding density maxima. The required penetration force across all specimens showed a large variation; the minimum force required to penetrate the SBP was $9 \mathrm{~N}$, the
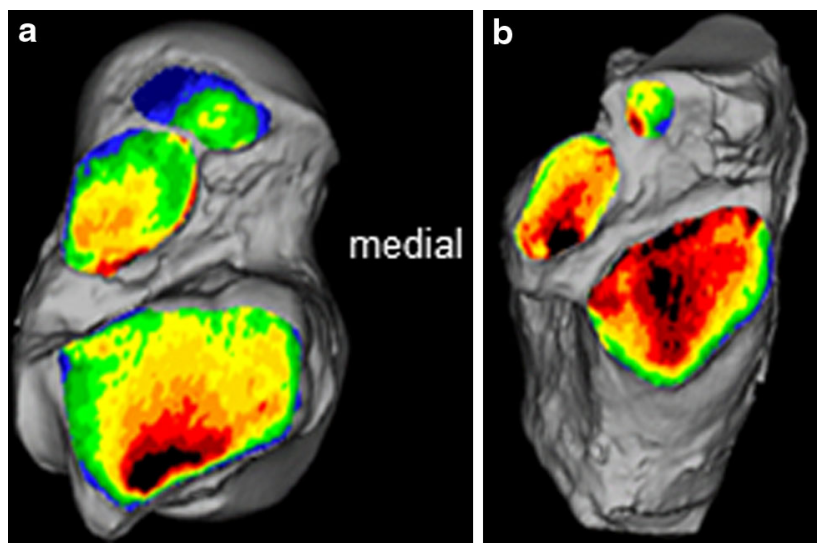

Fig. 4 Density distribution on one representative sample (sample 9). a Right talus, inferior aspect; $\mathbf{b}$ right calcaneus, superior aspect 
Fig. 5 Visual representation of density and penetration force (posterior talar articular surface of two calcanei). a, c Sample 1. b, d Sample 2. a, b Distribution pattern of density values. c, d Distribution pattern of penetration force values
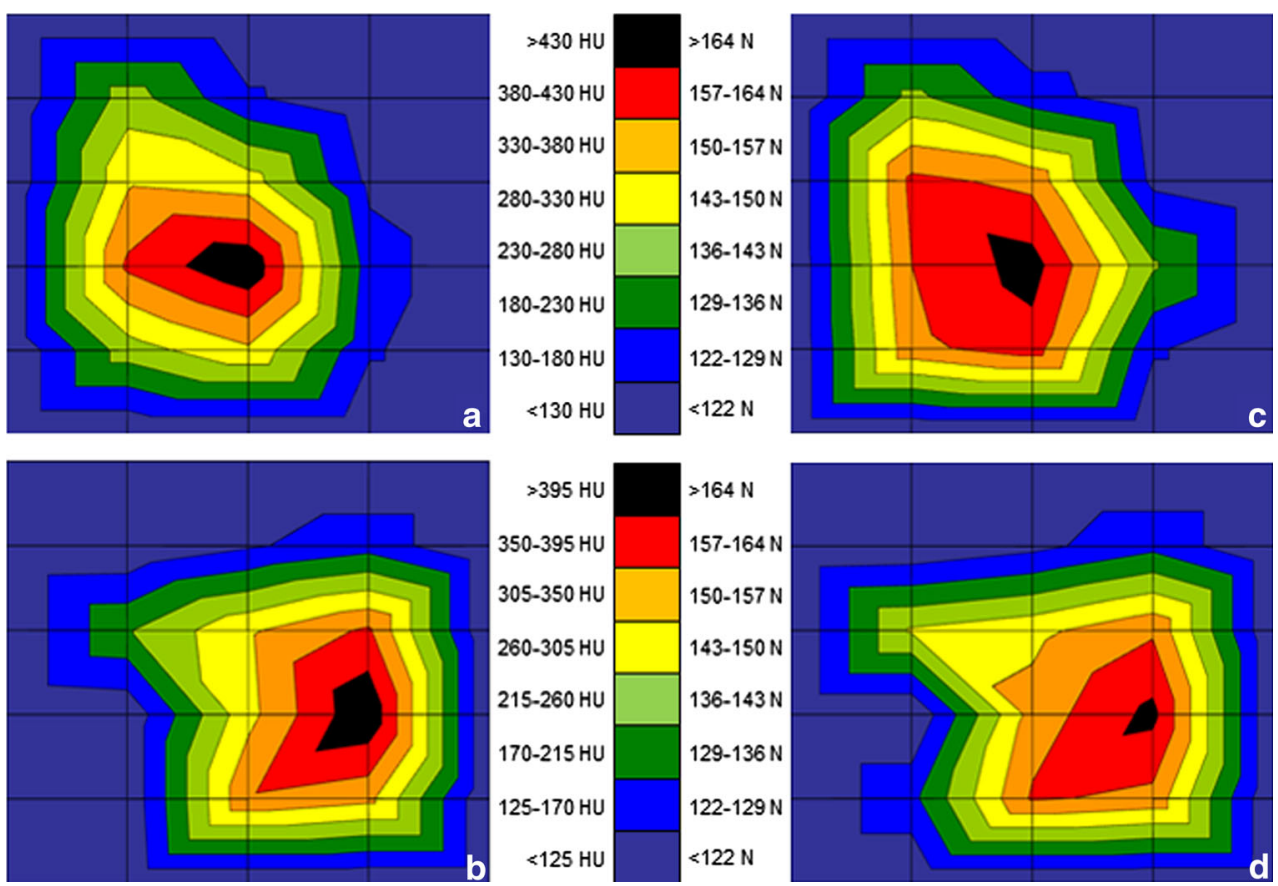

highest measured value was $1,269 \mathrm{~N}$ (mean $307 \mathrm{~N}$ ) (Table 1).

Density-strength correlation

By comparing the density distribution patterns and the distribution of penetration force visually, there could already be seen strong similarities (Fig. 5). The statistical evaluation of the value pairs of density and mechanical strength confirmed a linear dependence between density and strength values. The coefficient of correlation $\left(r^{2}\right)$ ranged from 0.76 to 0.95 (mean 0.88). Statistical significance $(p<0.01)$ was demonstrated in our study population (Table 1). A normal or log normal distribution of data could not be proved (Kolmogorov-Smirnov test).

\section{Discussion}

The present study describes (a) the anatomy and (b) the structure of the SBP of 21 tali, calcanei and navicular bones of European adults using the native samples for investigation of anatomical landmarks and classification as well as IT, and conventional CT-datasets for CT-OAM.

According to Bunning's [7] classification of European calcanei, we found Type A dominant in our study population with over $81 \%$. Type B showed an occurrence of $19 \%$, Type C was not seen at all as it is common for Europeans [5]. Overall, our samples, therefore, represent regular European distribution of the arrangement of the articular surfaces (Table 2).
First investigations of our samples determined in addition a high variability of the middle and anterior articular surface. To describe the bones, especially the calcanei, more precisely, we used the advanced calcanei classification established by Sharada et al. [26]. Here, the configuration of the anterior and middle articular surface becomes centre of interest. The general distribution of our samples following Sharada's classification differed quite severely from their data. Like Sharada et al. explained, this is mainly due to differences in race. Despite our small amount of samples, Table 2 gives a good impression of how European samples are distributed among Sharada's classification.

Following recent derived data on classification of the configuration of the posterior subtalar joint according to Shahabpour et al. [25] who analysed European samples of the talocalcaneal joint, our sample distribution matches their data (Table 2). Landmarks and anatomical structures described by them on thin-sliced MRI-data could be seen in our CT-datasets as well and revealed a similar sample distribution.

Following the anatomical analysis towards the microanatomy, we experimentally determined the mineralisation distribution of the SBP of the classified samples as a correlate of long-term stress in the posterior subtalar and the talonavicular joint and described the relationship between SBP density and mechanical strength using CT-OAM (for density analysis regarding to the mineralisation) and IT (for mechanical strength analysis).

Previously, several studies have investigated the load on these two joints but mainly focused on static and short-term 
conditions $[14,17,21,23,30]$. By means of pressuresensitive film the instantaneous load on the articular surfaces was determined. However, the results reported by these studies are contradictory. For the subtalar joint (especially the posterior articular surfaces), Mulcahy described a bicentric distribution of the main contact zones, located anterolaterally and posteromedially [17]. Essentially, Wagner and Reeck came to the same conclusion [21, 30]. In contrast to these results, Seangeorzan's [23] study also showed a bicentric distribution of high pressure zones but they were located in the anteromedial and posterolateral portions of the articular surfaces. Akiyama et al. [2] proved in a more recent work that there is a significant correlation of cartilage thickness and SBP density. Using 3D-CT to create bone surface models and a 3D-digitizer to create cartilage surface models, it was shown that cartilage tended to be the thickest in the areas where the SBP is most dense. Only investigating the posterior articular surfaces of the subtalar joint, their results revealed that in the posterior calcaneal articular surface of the tali the areas of greatest cartilage thickness could be found in the centre. In the posterior talar articular surface of the calcanei the cartilage thickness was more uniform with no clear maximum. These findings lead to the assumption that subchondral bone density should be highest in the central region of the posterior calcaneal articular surface of the tali and that there should be found no clear density maximum in the posterior talar articular surface of the calcanei.

In the present study, most of the posterior talar articular surfaces of the calcanei revealed a monocentric pattern ( $n=13$; localisation: central). Only a minority of the samples showed bicentric maxima ( $n=4$; localisation: anterior and medial) or even a tricentric pattern ( $n=4$; localisation: anterior, posterior, and medial). The posterior calcaneal articular surface of the tali showed monocentric $(n=16$; localisation: central or lateral) or bicentric $(n=5$; localisation: posteromedial and lateral). Comparing our results with those of the above-mentioned studies, several differences become apparent. First, high pressure zones, which can be regarded as a correlate of short-term stress, always showed a bicentric distribution, whereas the mineralisation patterns of the SBP mostly presented a monocentric distribution which can be regarded as a correlate to long-term load intake. Second, the mineralisation patterns are in good agreement with the results of Akiyama et al. concerning the posterior calcaneal articular surface of the tali. Although clear density maxima could be found in the posterior talar articular surface of the calcanei, these results support the findings of Akiyama et al. [2] concerning a rather uniform density distribution since the range between the highest and lowest density values was significantly larger in the talar part (average range 85 8-bit $\mathrm{HU}$ ) of the subtalar joint than in the calcaneal part (average range 53.8 8-bit HU).
In the talonavicular joint, the works of Beaudoin, Mall/ Koebke, and Reeck [6, 14, 21] have yielded contradictory results as well. Beaudoin and Mall/Koebke $[6,14]$ described a high pressure zone located in the dorsolateral portion, though Reeck [21] found pressure maxima either located around the edges of the articular surfaces or in a narrow region in the centre.

In the present study, the density distribution in the navicular articular surface of the tali mostly showed a monocentric pattern with dorsolaterally located maxima. The majority of the talar articular surfaces of the navicular bone also showed monocentric distribution patterns, situated dorsally or dorsolaterally. Consequently, these results coincide with those from the works of Beaudoin and Mall/ Koebke [6, 14].

In terms of a possible correlation between the mineralisation and the mechanical strength of the SBP it can be said that the results of the present study are in good agreement with previous studies dealing with the same question on other joints $[10,16]$. The maximal required force to penetrate the SBP was located in the areas, where the SBP was most dense. The average coefficient of correlation was 0.88 which can be regarded as a strong association of mineralisation and mechanical strength.

The results showed that the increase in mechanical strength per density unit was less significant in specimens of low average mineralisation than in highly mineralised specimens (Fig. 6). This leads to the assumption that other factors apart from the concentration of calcium hydroxyl apatite play a role in increasing the mechanical strength of the SBP as only $75-80 \%$ of bone strength is attributed to this system [27]. In the SBP itself, the mechanical properties are additionally influenced by the collagenous structure. The composition and degree of collagen crosslinking can increase the mechanical strength of the SBP as well since the helices of bone's type I collagen are hereby fastened together [24]. The underlying trabecular bone is an important factor concerning the stabilisation of the SBP. Because the connectivity and structure of the trabecular bone is known to be involved in the modification of bone strength and the support of the SBP, it might be possible that after adapting the SBP to its maximum strength by calcium deposition and collagen cross-linking a further increase of bone strength may be achieved by remodelling the trabecular structure into a more stabilising arrangement and supporting the SBP from beneath [4].

Potential limitations of the present study and the used methods are mainly due to the complex structure of the tested specimens. Despite all effort to perform the IT with maximum accuracy, deviations regarding the perpendicular orientation of the intender on the investigated surface had to be accepted. A second parameter that possibly influenced the results is the preservation of the tested specimens 
Fig. 6 Comparison of correlation between mineral density and mechanical strength of two representative samples. a High average load tolerance (sample $1, r^{2}=0.84$ ). b Low average load tolerance (sample $\left.2, r^{2}=0.86\right)$
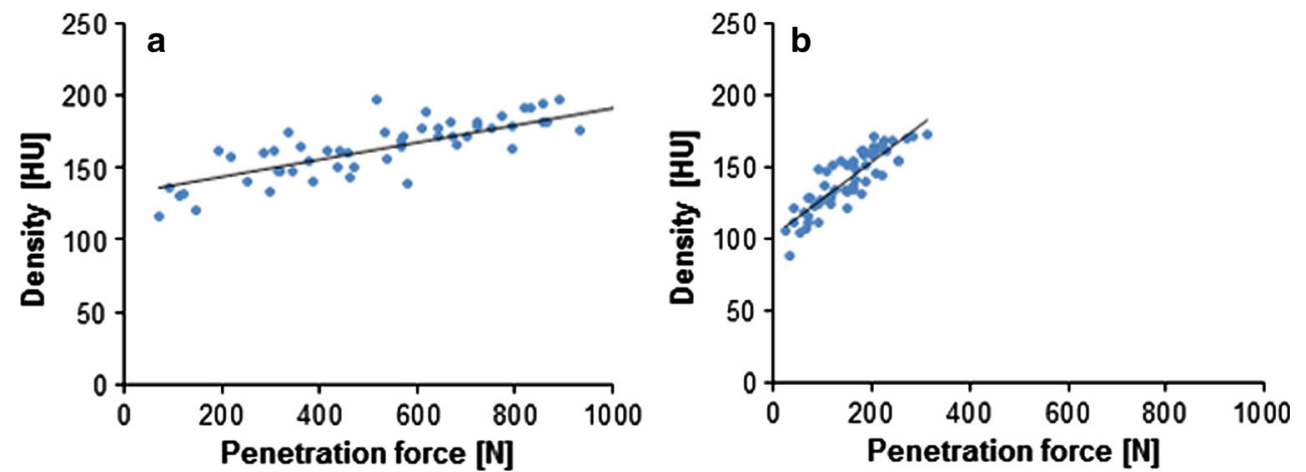

as they were fixated in formalin. In the literature, Burkhart et al. and Van Haaren et al. [8, 29] reported that formalin fixation had no measurable effect on bone mineral density or mechanical strength compared to fresh specimens. In contrast, there is a discussed decrease in plastic energy absorption due to formalin fixation tested on cortical specimens of the human femur [28]. Since the present study mainly investigated the correlation between mineralisation and mechanical strength of the SBP and absolute values were not used for comparison, the fixation method is not of critical importance.

Due to the confirmed correlation between the density, respectively the mineralisation, and the mechanical strength of the SBP it can be concluded that CT-OAM can be used to indirectly gain information about the mechanical strength of a joint. In addition, the collected data could help understanding changes in the mechanics of the investigated joints and their long-term effects which are important to be known in pathological states and after performed surgical corrections. For example, long-term changes in joint mechanics that can be seen after lateral opening wedge osteotomy [3] could be visualized and monitored. This could help to improve postsurgical treatment and outcome.

Acknowledgments We would like to thank Mrs. Mireille Toranelli for the software-support and Mrs. Nicole Hauser for her help with management of CT-data. Furthermore, the authors received no grant or sources of financial support related to the topic or topics of this article.

Conflict of interest The authors declare that they have no conflict of interest.

\section{References}

1. Aitken GK, Bourne RB, Finlay JB, Rorabeck CH, Andreae PR (1985) Indentation stiffness of the cancellous bone in the distal human tibia. Clin Orthop Relat Res 201:264-270

2. Akiyama K, Sakai T, Sugimoto N, Yoshikawa H, Sugamoto K (2012) Three-dimensional distribution of articular cartilage thickness in the elderly talus and calcaneus analyzing the subchondral bone plate density. Osteoarthritis Cartilage 20(4):296-304. doi:10.1016/j.joca.2011.12.014

3. Aly T (2011) Management of valgus extra-articular calcaneus fracture malunions with a lateral opening wedge osteotomy. J Foot Ankle Surg 50(6):703-706. doi:10.1053/j.jfas.2011.04.006

4. Ammann P, Rizzoli R (2003) Bone strength and its determinants. Osteoporos Int 14(Suppl 3):S13-S18. doi:10.1007/s00198-0021345-4

5. Barbaix E, Van Roy P, Clarys JP (2000) Variations of anatomical elements contributing to subtalar joint stability: intrinsic risk factors for post-traumatic lateral instability of the ankle? Ergonomics 43(10):1718-1725. doi:10.1080/001401300750004122

6. Beaudoin AJ, Fiore SM, Krause WR, Adelaar RS (1991) Effect of isolated talocalcaneal fusion on contact in the ankle and talonavicular joints. Foot Ankle 12(1):19-25

7. Bunning PS, Barnett CH (1965) A comparison of adult and foetal talocalcaneal articulations. J Anat 99:71-76

8. Burkhart KJ, Nowak TE, Blum J, Kuhn S, Welker M, Sternstein W, Mueller LP, Rommens PM (2010) Influence of formalin fixation on the biomechanical properties of human diaphyseal bone. Biomed Tech (Berl) 55(6):361-365. doi:10.1515/BMT.2010.043

9. Chen YX, Yu GR, Mei J, Zhou JQ, Wang W (2008) Assessment of subtalar joint neutral position: a cadaveric study. Chin Med J (Engl) 121(8):735-739

10. Hoechel S, Wirz D, Muller-Gerbl M (2012) Density and strength distribution in the human subchondral bone plate of the patella. Int Orthop 36(9):1827-1834. doi:10.1007/s00264-012-1545-2

11. Hofmann AA, Hammon DJ, Daniels AU (1991) Compressive strength mapping of femoral head trabecular bone. J Rehabil Res Dev 28(2):25-32

12. Madry H, van Dijk CN, Mueller-Gerbl M (2010) The basic science of the subchondral bone. Knee Surg Sports Traumatol Arthrosc 18(4):419-433. doi:10.1007/s00167-010-1054-z

13. Mahjoub M, Berenbaum F, Houard X (2012) Why subchondral bone in osteoarthritis? The importance of the cartilage bone interface in osteoarthritis. Osteoporos Int 23(Suppl 8):841-846. doi:10.1007/s00198-012-2161-0

14. Mall G, Koebke J (1993) Stress fractures of the navicular bonebiomechanical and densitometry studies. Sportverletz Sportschaden 7(2):73-77. doi:10.1055/s-2007-993486

15. Milz S, Eckstein F, Putz R (1997) Thickness distribution of the subchondral mineralization zone of the trochlear notch and its correlation with the cartilage thickness: an expression of functional adaptation to mechanical stress acting on the humeroulnar joint? Anat Rec 248(2):189-197

16. Muhlhofer H, Ercan Y, Drews S, Matsuura M, Meissner J, Linsenmaier U, Putz R, Muller-Gerbl M (2009) Mineralisation and mechanical strength of the subchondral bone plate of the inferior tibial facies. Surg Radiol Anat 31(4):237-243. doi:10. 1007/s00276-008-0430-6 
17. Mulcahy DM, McCormack DM, Stephens MM (1998) Intraarticular calcaneal fractures: effect of open reduction and internal fixation on the contact characteristics of the subtalar joint. Foot Ankle Int 19(12):842-848

18. Muller-Gerbl M (1998) The subchondral bone plate. Adv Anat Embryol Cell Biol 141:III-XI, 1-134

19. Perry J (1983) Anatomy and biomechanics of the hindfoot. Clin Orthop Relat Res 177:9-15

20. Radin EL, Rose RM (1986) Role of subchondral bone in the initiation and progression of cartilage damage. Clin Orthop Relat Res 213:34-40

21. Reeck J, Felten N, McCormack AP, Kiser P, Tencer AF, Sangeorzan BJ (1998) Support of the talus: a biomechanical investigation of the contributions of the talonavicular and talocalcaneal joints, and the superomedial calcaneonavicular ligament. Foot Ankle Int 19(10):674-682

22. Saitoh S, Nakatsuchi Y, Latta L, Milne E (1994) Distribution of bone mineral density and bone strength of the proximal humerus. J Shoulder Elbow Surg 3(4):234-242. doi:10.1016/S10582746(09)80041-4

23. Sangeorzan BJ, Wagner UA, Harrington RM, Tencer AF (1992) Contact characteristics of the subtalar joint: the effect of talar neck misalignment. J Orthop Res 10(4):544-551. doi:10.1002/jor. 1100100409

24. Seeman E, Delmas PD (2006) Bone quality - the material and structural basis of bone strength and fragility. N Engl J Med 354(21):2250-2261. doi:10.1056/NEJMra053077
25. Shahabpour M, Deville A, Van Roy P, Vaes P, De Mey J, De Maeseneer M (2011) Magnetic resonance imaging of anatomical variants of the subtalar joint. Surg Radiol Anat 33(7):623-630. doi:10.1007/s00276-011-0788-8

26. Sharada R, Sneha K, Gupta C, Pai SR, Rairam GB (2012) Nonmetrical study of the pattern of talar articular facets in south Indian dry calcanei. Surg Radiol Anat 34(6):487-491. doi:10. 1007/s00276-012-0939-6

27. Smith CB, Smith DA (1976) Relations between age, mineral density and mechanical properties of human femoral compacta. Acta Orthop Scand 47(5):496-502

28. Unger S, Blauth M, Schmoelz W (2010) Effects of three different preservation methods on the mechanical properties of human and bovine cortical bone. Bone 47(6):1048-1053. doi:10.1016/j.bone. 2010.08 .012

29. van Haaren EH, van der Zwaard BC, van der Veen AJ, Heyligers IC, Wuisman PI, Smit TH (2008) Effect of long-term preservation on the mechanical properties of cortical bone in goats. Acta Orthop 79(5):708-716. doi:10.1080/17453670810016759

30. Wagner UA, Sangeorzan BJ, Harrington RM, Tencer AF (1992) Contact characteristics of the subtalar joint: load distribution between the anterior and posterior facets. J Orthop Res 10(4):535-543. doi:10.1002/jor.1100100408 